\title{
Industry and Academic Medicine: A Dangerous Liaison?
}

The pharmaceutical industry has become increasingly powerful in the conduct of clinical trials, evolving from simple, basic funding to detailed involvement in the scientific design and overall supervision of large multicentre trials. National and international clinicians and scientists have been recruited to design and chair these studies, in close liaison with industry, but their neutrality is under scrutiny and is frequently questioned by the academic community.

There is strong evidence that policy statements concerning new drugs, and clinical practice guidelines drawn up by select committees are often influenced by industry, resulting in major financial expenditure on drugs often of marginal value over cheaper, simpler alternatives.

The relationship between the medical profession and pharmaceutical industry is rapidly evolving, molded by the forces of scientific rigor and the increasingly demanding standards of government and other funding agencies. Both sides are now mutually dependent, so a compromise must emerge to the benefit of the patients.

Rarely does a week pass without an article or commentary in a major medical journal critical of the increasing impact of the drug industry on the practice of clinical medicine. The dramatic expansion of medical knowledge over the last decades, coupled with an increasingly informed and sophisticated public, has proven a mixed blessing. In spite of the new religion of evidencebased medicine, charlatanry remains both rampant and lucrative and, even today, some of the drugs widely used for the treatment or prevention of stroke throughout the world are of dubious scientific validity.

The conduct of clinical trials has become considerably sophisticated over the years. Initially, representatives from the drug industry approached individual investigators at random to perform a preplanned study, nominated one investigator as the first author, and maximized any favourable conclusions, often based on doubtful statistics and vanishingly small numbers of patients. With increasingly sophisticated methodology and more stringent and demanding guidelines by government agencies such as the US Food and Drug Administration and the Canadian Health Protection Branch, a resourceful pharmaceutical industry began to employ well-established academics, who designed drug trials with better science and more adequate numbers of patients, and whose prestige could attract investigators nationwide or even worldwide. However, busy academic investigators often proved poor recruiters, delegating the critical task of accurate data recording to overcommitted or disinterested junior staff. More informed investigators questioned trial methodology and demanded access to the data for their own independent scrutiny and analysis.

Industry responded with the emergence of Contract Research Organizations (CROs), business companies that contract with industry to carry out clinical trials for a price and with a deadline, firing unproductive centres irrespective of their academic pedigree. Their primary goal is profit, not scientific information. They often enroll community physicians with no expertise in the subject under study, nor particular interest in subsequent publications but who, for a price, can quickly recruit large numbers of appropriate patients. However, they also play a positive role, since they are often more fastidious with data management than the investigator, insisting on fulfillment of all details of inclusion and exclusion criteria and other critical data entry points.

In an attempt to avoid the 'divide and conquer' strategy of industry, neurologists in Canada set up a consortium of stroke investigators to vet any drug trial proposed by industry, and participate only in those stroke trials that they felt had a potentially practical and scientific basis. Gradually this organization has involved most stroke neurologists, so most stroke trials can only be performed with the consortium's agreement. Nevertheless, the consortium remains subject to all the guarded and covert restrictions of industry, with difficulty of access to data and limited independent right of publication.

However, industry can marshal financial resources rarely matched by independent granting agencies, and drug companies now employ their own armies of well-trained statisticians, epidemiologists and other personnel needed to produce the detailed and exact data necessary to meet government standards of safety and efficacy for release of the drug to the public. In spite of these safeguards, the safety and efficacy of approved drugs are still questioned, because of genuine concern in the medical profession, the public and the media about the coziness between pharmaceutical companies and academic medicine. The impact of this climate of suspicion was recently demonstrated in the controversy over thrombolytic treatment for acute ischemic stroke. A nonmedical investigative journalist alleged in the British Medical Journal that the acceptance by a consensus committee of the published results of a thrombolytic trial demonstrating therapeutic effectiveness was influenced by gifts from industry to the American Heart Association amounting to over $\$ 11$ million. ${ }^{1}$ The article insinuated guilt by association but failed to prove more than this. However, yet another signal was sent to academia to tighten the rules of this association.

Physicians are viewed with mistrust unless they remain more than "arms length" from the temptations offered by industry for their compliance, the benefits ranging from minor gifts such as tee-shirts to major benefits such as paid luxury vacations. Few busy clinicians have time to critically appraise original articles and so depend on reviews by more informed colleagues. These influences on their prescribing habits are often subliminal and accepted uncritically. ${ }^{2}$ In one survey, most medical students disapproved of politicians accepting 'gifts', yet few felt it improper to accept gifts of similar value from pharmaceutical companies. ${ }^{3}$ Following a MEDLINE search designed to evaluate 
the interrelationship between industry and the medical profession, Wazana ${ }^{3}$ warned of the growing influence of pharmaceutical marketing on prescribing practice. The young physician, faced with the appropriate patient, will reflexly prescribe a 'better' drug than the standard preparation, based on a recent recommendation from an academic leader or consensus panel. These 'me too' drugs often differ only in their exorbitant price rather than improved and often marginal efficacy.

Another powerful factor influencing physicians' prescribing habits is the effect of Clinical Practice Guidelines. Usually these recommendations for optimal clinical practice are devised by a committee of established and experienced academic clinicians, appointed by a recognized authority such as the Academy of Neurology or American Heart Association, and the subsequent publications are subject to peer review. Who better to evaluate the evidence objectively and make recommendations? However, sometimes industry will sponsor similar meetings, and their recommendations, often published as supplements in specialty journals, are used as reference bases, but without the rigor of peer review needed for publication in leading medical journals.

Scientific conferences may have specially assigned sessions sponsored by industry where practice guidelines are presented and debated, sometimes with attempts to influence the proceedings in favour of industry. In a survey of 44 clinical practice guidelines, $52 \%$ of the authors replied to the questionnaire, and there was evidence of 'considerable interaction' between the authors and industry, indicating serious bias in favour of the drug, prompting a call for a formal process to assess conflict of interest. ${ }^{4}$

To neutralize these influences, the policies of many medical journals have become increasingly rigorous in assuring that published articles or reviews come under no undue commercial pressure, and demanding disclosure of any financial associations of authors. Just when the commercial pressure is significant is more difficult to define; trivial or minor gifts are unlikely to sway an authors judgment, in accord with the policies of the National Institutes of Health and Association of American Medical Colleges. ${ }^{5}$ Relman ${ }^{6}$ has questioned whether honoraria of $\$ 10,000$ per annum or industrial research support of up to 10 to $20 \%$ of a research programme can really be considered 'trivial' and recommends prohibition of any level of support, rather than devising an arbitrary 'negotiable'level.

However, government and charitable foundations alone cannot support the enormous expenditures needed today in financing clinical research. At present, the needs of academic medicine and industry are reciprocal and interdependent. Some of the newer effective AIDS drugs could not have been developed without industry support ${ }^{7}$ so it is important that the profit motive does not invalidate scientific excellence. This will be to the advantage of both parties. The secret is to find equipoise between the needs of the company and those of the investigators, yet still maintaining their academic independence. Bodenheimer ${ }^{8}$ believes that while drug trials should be funded by industry, the design, implementation, data analysis and publication should be controlled entirely by the medical profession, but would industry share this unquestioned trust? Until contracts between academic medicine and industry guarantee unlimited access to primary data throughout the course of the entire study, and total independence in publishing the data, ${ }^{9}$ we remain under a cloud of suspicion from our colleagues, yet at the same time enduring the frustrations of being investigators for hire.

Academic medicine, assisted by industry, must play a more pro-active role in evaluating the accuracy, applicability, cost effectiveness and limitations of new drugs and devices. The education of the practitioner about these new treatments should be the responsibility of unbiased and critical academic physicians with public declarations of neutrality. At present, academic medicine maintains an unstable liaison with industry, but this is still evolving. Both sides know they need each other and, in fact, cannot survive in the present economic climate without each other. A solution must be found, without recriminations to industry or accusations of conflict of interest from our colleagues. The only solution is to work together to the benefit not just of the parties concerned, but more important, to our patients.

John W Norris

Toronto, Ontario

\section{ACKNOWLEDGEMENT}

I thank Dr Henry J. M. Barnett for his helpful advice and criticism. Dr Norris is a member of the Data Safety Monitoring Board of ONO USA. The views expressed are solely those of the author and do not necessarily represent those of the Journal.

\section{REFERENCES}

1. Lenzer J. Alteplase for stroke: money and optimistic claims buttress the brain attack: campaign. Br Med J 2002;324:723-729.

2. Editorial. The invisible hand of the marketing department. CMAJ 2002;167:5.

3. Wazana A. Physicians and the pharmaceutical industry. JAMA 2000;283:373-380.

4. Choudhry NK, Stelfox HT, Detsky AS. Relationships between authors of clinical practice guidelines and the pharmaceutical industry. JAMA2002;287:612-617.

5. Drazen JM, Curfman GD. Financial associations of authors. N Engl J Med 2002;346:1901-1902.

6. Relman AS. Financial associations of authors. N Engl J Med 2002;347:1043

7. Montaner JSG, O'Shaughnessy MV, Schechter MT. Industrysponsored clinical research: a double-edged sword. Lancet 2001;358:1893-1894.

8. Bodenheimer T. Uneasy alliance-clinical investigators and the pharmaceutical industry. N Engl J Med 2000;324:1539-1544.

9. Drazen JM. Institutions, contracts, and academic freedom. N Engl J Med 2002;347:1362-1363. 